UW/PT 99-25

\title{
Non-perturbative dynamics of hot non-Abelian gauge fields: beyond leading log approximation
}

\author{
Peter Arnold \\ Department of Physics, University of Virginia, Charlottesville, VA 22901 \\ Laurence G. Yaffe \\ Department of Physics, University of Washington, Seattle, Washington 98195
}

(December 1999)

\begin{abstract}
Many aspects of high-temperature gauge theories, such as the electroweak baryon number violation rate, color conductivity, and the hard gluon damping rate, have previously been understood only at leading logarithmic order (that is, neglecting effects suppressed only by an inverse logarithm of the gauge coupling). We discuss how to systematically go beyond leading logarithmic order in the analysis of physical quantities. Specifically, we extend to next-to-leading-log order (NLLO) the simple leading-log effective theory due to Bödeker that describes non-perturbative color physics in hot non-Abelian plasmas. A suitable scaling analysis is used to show that no new operators enter the effective theory at next-to-leading-log order. However, a NLLO calculation of the color conductivity is required, and we report the resulting value. Our NLLO result for the color conductivity can be trivially combined with previous numerical work by G. Moore to yield a NLLO result for the hot electroweak baryon number violation rate.
\end{abstract}

\section{INTRODUCTION}

The near equilibrium dynamics of hot, weakly-coupled non-Abelian plasmas, such as high temperature QCD or electroweak theory, involves a surprisingly rich hierarchy of spatial and temporal scales. Of particular interest in recent years is the analysis of non-perturbative processes occurring in (or near) equilibrium in such plasmas. A primary application is to the rate of baryon number violation in hot electroweak theory, which quite possibly is responsible for the preponderance of matter over anti-matter in our universe [1]. Baryon number nonconservation is possible due to the electroweak anomaly in the baryon (and lepton) number current, and proceeds through non-perturbatively large thermal fluctuations in electroweak gauge fields. A key goal is to understand how to calculate the dynamics of such fluctuations.

As will be reviewed momentarily, non-perturbative gauge dynamics in hot non-Abelian plasmas may be described (at leading logarithmic order) by a remarkably simple effective 
theory discovered by Bödeker [2], whose only input parameter is the value of the color ${ }^{1}$ analog of electrical conductivity. The color conductivity in turn depends on what is known as the hard gauge boson damping rate, which is a measure of the rate at which collisions randomize a charge carrier's color charge. Up to now, however, these quantities have only been known at leading order in the logarithm of the gauge coupling; that is, neglecting effects suppressed only by $1 / \ln (1 / g)$, where $g$ is the gauge coupling. Consequently, numerical simulations of the high temperature topological transition (or baryon number violation) rate based on Bödeker's effective theory have only been valid to leading order in logarithms [3]. For practical purposes such leading log results are, by themselves, not very useful - there is a huge difference between, say, $\ln (16 \pi / g)$ and $\ln (1 / 10 g)$ for any realistic value of the gauge coupling. But this difference is of sub-leading order in $\ln (1 / g)$. In this paper, we report a calculation of the color conductivity to next-to-leading-log order (NLLO), and in the process explain what this means in gauge-invariant language. Our approach involves systematically constructing a sequence of effective theories which reach from short to long distance scales, and performing perturbative calculations which enable one to match the physics of each effective theory with its predecessor to the order desired. When combined with Moore's numerical result [3] for the constant of proportionality between the topological transition rate and the color conductivity, our improved value for the conductivity immediately yields a NLLO result for the topological transition rate.

In this paper, "hot" plasma means hot enough (1) to be ultra-relativistic, (2) to ignore chemical potentials, (3) for non-Abelian gauge couplings to be small, and (4) to be in the high temperature symmetric phase. ${ }^{2}$ For convenient reference, the critical distance and time scales associated with color dynamics in such plasmas (to be reviewed below) are collected in table I, and the notation used in this paper is summarized in table II.

\begin{tabular}{ll}
\hline$O(T)$ & typical momentum of excitations in the plasma. \\
$m=O(g T)$ & inverse Debye screening length. \\
$\gamma=O\left[g^{2} T \ln \left(g^{-1}\right)\right]$ & hard gauge boson damping rate. \\
$O\left(g^{2} T\right)$ & inverse spatial scale of non-perturbative gauge fluctuations. \\
$O\left[g^{4} T \ln \left(g^{-1}\right)\right]$ & inverse time scale of non-perturbative gauge fluctuations. \\
\hline
\end{tabular}

TABLE I. Important (inverse) distance and time scales.

\footnotetext{
${ }^{1}$ We use "color" as a generic term for some non-Abelian gauge charge, not as something specific to QCD.

${ }^{2}$ We use the term "symmetric phase" loosely since, depending on the details of the Higgs sector, there need not be any sharp transition between the symmetric and "symmetry-broken" phases of the theory [4]. A sharp transition is required for viable electroweak baryogenesis. Our analysis is applicable whenever the infrared dynamics of the Higgs field is irrelevant at lengths of $O\left(1 / g^{2} T\right)$, which is the case either (a) far above the electroweak phase transition or "crossover," or (b) in the symmetric phase at the transition in cases where there is a sufficiently strong first-order transition.
} 
$\overline{v^{\mu}}=(1, \mathbf{v}) ; \mathbf{v}$ a spatial unit vector.

$\mathbf{A}=\mathbf{A}(\mathbf{x}, t)$, the spatial non-Abelian gauge field.

$\mathbf{D}=\boldsymbol{\nabla}+g \mathbf{A}^{a} T^{a}$, the gauge covariant derivative.

$W=W(\mathbf{x}, \mathbf{v}, t)$, the adjoint color distribution of hard excitations.

$\boldsymbol{\zeta}=\boldsymbol{\zeta}(\mathbf{x}, t)$ and $\xi=\xi(\mathbf{x}, \mathbf{v}, t)$ are Gaussian white noise.

$\langle\langle\cdots\rangle\rangle$ denotes averaging over noise.

$\langle\cdots\rangle \equiv\langle\cdots\rangle_{\mathbf{v}}$ denotes averaging over the direction $\mathbf{v}$.

$\delta^{S_{2}}\left(\mathbf{v}-\mathbf{v}^{\prime}\right)$ is a $\delta$-function on the two-sphere normalized so that $\left\langle\delta^{S_{2}}\left(\mathbf{v}-\mathbf{v}^{\prime}\right)\right\rangle_{\mathbf{v}^{\prime}}=1$.

$\delta \hat{C} W \equiv\left\langle\delta C\left(\mathbf{v}, \mathbf{v}^{\prime}\right) W\left(\mathbf{v}^{\prime}\right)\right\rangle_{\mathbf{v}^{\prime}}$, the linearized collision operator applied to $W$.

$C_{\mathrm{A}}$ is the adjoint Casimir of the gauge group $[N$ for $\mathrm{SU}(N)]$.

$d=3-\epsilon$ with $\epsilon \rightarrow 0$, the number of spatial dimensions.

TABLE II. Summary of notation.

\section{REVIEW}

It is well known that non-perturbative gauge field fluctuations in hot non-Abelian plasmas are essentially magnetic and characterized by the distance scale ${ }^{3} R \sim\left(g^{2} T\right)^{-1}$. The corresponding time scale has only been understood more recently, and can be arrived at by the following simple physical argument. Start with (non-Abelian) Ampere's Law, $\mathbf{D} \times \mathbf{B}=D_{t} \mathbf{E}+\mathbf{j}$. Now recall that plasmas are conductors, and so write $\mathbf{j}=\sigma \mathbf{E}$, where $\sigma$ is the (color) conductivity. If we assume the time dependence will be slow and treat the covariant time derivative $D_{t} \mathbf{E}$ as negligible compared to $\sigma \mathbf{E}$ (an approximation that can be verified a posteriori), then the result is

$$
\mathbf{D} \times \mathbf{B}=\sigma \mathbf{E}
$$

Extracting the time scale from this equation is clearest if one specializes to $A_{0}=0$ gauge:

$$
\mathbf{D} \times \mathbf{D} \times \mathbf{A}=\sigma \frac{d}{d t} \mathbf{A}
$$

In terms of characteristic scales, this is just

$$
R^{-2} \mathbf{A} \sim \sigma t^{-1} \mathbf{A}
$$

and so the characteristic time $t \sim \sigma R^{2} \sim \sigma / g^{4} T^{2}$. The color conductivity $\sigma$ was originally analyzed by Selikhov and Gyulassy [7] and is order $\sigma \sim T / \ln (1 / g)$, yielding $t \sim\left[g^{4} T \ln (1 / g)\right]^{-1}$. The rate per unit volume $\Gamma$ for topological transitions in a hot, non-Abelian plasma must therefore have the characteristic size $\Gamma \sim 1 /\left(R^{3} t\right) \sim \alpha^{5} T^{4} \ln (1 / \alpha)$, where as usual $\alpha \equiv g^{2} / 4 \pi$. The factor of $\alpha^{5}$ in this rate was originally noted by ourselves and Son [8], and the presence of the logarithm by Bödeker [2]. The explanation in terms of color conductivity is given in detail in Ref. [5].

\footnotetext{
${ }^{3}$ See the introduction of Ref. [5] for a simple heuristic argument, or Ref. [6] for more formal ones.
} 
The above order of magnitude arguments can be made quantitative. One can numerically integrate, with Boltzmann-weighted initial conditions, the classical equation (2) that we derived from Ampere's Law, and measure the frequency of topological transitions [3]. A classical, as opposed to quantum, treatment is adequate because the low momentum modes $(k \ll T)$ of the gauge field in a hot plasma have large occupation numbers $\left[n_{k} \sim\left(e^{k / T}-1\right)^{-1} \sim T / k \gg 1\right]$ and so, by the correspondence principle, behave classically [9]. Corrections to the classical approximation are suppressed by powers of $k / T$. In our case, we are interested in modes with $k \sim R^{-1} \sim g^{2} T$, which is indeed small compared to $T$ for weak coupling.

However, Eq. (2) first requires some refinement. It is a purely dissipative equation. An effective theory for the equilibrium dynamics of the system must therefore incorporate a source of thermal noise to maintain thermal equilibrium. Ultimately, this noise is (like the dissipation) due to interactions of the modes of interest with other degrees of freedom of the system, specifically typical charge carriers, which have momentum of order $T$. As discussed in Ref. [5], one may argue from general principles that, at the distance and time scales of interest, this noise can be taken to be simple Gaussian white noise, appropriately normalized to produce the desired temperature $T$. The resulting effective theory is ${ }^{4}$

$$
\mathbf{D} \times \mathbf{B}+\boldsymbol{\zeta}=\sigma \mathbf{E},
$$

where $\boldsymbol{\zeta}$ is Gaussian noise with correlation

$$
\left\langle\left\langle\zeta_{i}(t, \mathbf{x}) \zeta_{j}\left(t^{\prime}, \mathbf{x}^{\prime}\right)\right\rangle\right\rangle=2 \sigma T \delta_{i j} \delta\left(t-t^{\prime}\right) \delta^{(3)}\left(\mathbf{x}-\mathbf{x}^{\prime}\right) .
$$

The effective theory (4) is also known as stochastic 3-dimensional gauge theory, and has a long history of theoretical study motivated by formal questions divorced from any connection with high temperature dynamics [14]. A key property of this theory is the fact that it is UV finite [5].

The final ingredient needed to make the effective theory quantitative, is the precise value of the color conductivity $\sigma$. This has previously been known only at leading log order $[7,2,5]$ :

$$
\sigma \approx \frac{m^{2}}{3 \gamma}
$$

where $\approx$ denotes equality up to relative corrections suppressed by $[\ln (1 / g)]^{-1}$. Here, $m$ is the leading-order Debye screening mass, which is well known, ${ }^{5}$ is $O(g T)$, and completely

\footnotetext{
${ }^{4}$ This equation certainly makes sense in $A_{0}=0$ gauge, and is also correct and unambiguous in general flow gauges of the form $A_{0}=R[\mathbf{A}]$, where $R[\mathbf{A}]$ depends on $\mathbf{A}(\mathbf{x}, t)$ only instantaneously and so does not involve time derivatives of A. See Ref. [10] for a discussion of flow gauges, and Ref. [11] for a proof that the equation (4) may be applied in any gauge of this class. There are subtleties in directly interpreting the Langevin equation (4) in other gauge choices, such as Landau gauge. For various historical reasons, our approach for doing actual calculations is to use a path integral representation of these Langevin equations, for which it is straightforward to fix any gauge desired using the usual Faddeev-Popov procedure. (See Ref. [12] for details.) We find it convenient, in particular, to use Coulomb gauge. But Coulomb gauge might also be implemented as the $\lambda \rightarrow \infty$ limit of the flow gauge $A_{0}=\lambda \boldsymbol{\nabla} \cdot \mathbf{A}$, for which there should be no problem working directly with the Langevin formulation (4) instead of the associated path integral representation.

${ }^{5}$ For $\mathrm{SU}(N)$ gauge theory with $N_{\mathrm{s}}$ scalars and $N_{\mathrm{f}}$ Dirac fermions in the fundamental representation, the Debye mass $m^{2}=\frac{1}{6}\left(2 N+N_{\mathrm{s}}+N_{\mathrm{f}}\right) g^{2} T^{2}$ at leading order in $g$. For hot electroweak theory with three fermion families and a single Higgs doublet, $m^{2}=\frac{11}{6} g^{2} T^{2}$.
} 
encapsulates the dependence on the matter field content of the underlying theory. $\gamma$ is the hard gauge boson damping rate [15] and, to leading-log order, is given by

$$
\gamma \approx C_{\mathrm{A}} \alpha T \ln \left(\frac{1}{g}\right) .
$$

The leading-log effective theory represented by (4) and (5) was first derived by Bödeker [2]. (For subsequent derivations, see Refs. [5,3,16-18].6) Using this theory, Moore [3] has numerically simulated the topological transition rate for electroweak theory, obtaining

$$
\Gamma \approx(10.8 \pm 0.7)\left(\frac{2 \pi}{3 \sigma}\right)(\alpha T)^{5}
$$

\section{THE SEQUENCE OF EFFECTIVE THEORIES}

The series of effective theories we will need to describe color dynamics at low frequency $\omega$ and momentum $k$ [ultimately $\omega \sim t^{-1} \sim\left[g^{4} T \ln (1 / g)\right]^{-1}$ and $\left.k \sim R^{-1} \sim\left(g^{2} T\right)^{-1}\right]$ are as follows.

Theory 1: $\omega, k \ll T$

$$
\begin{gathered}
\left(D_{t}+\mathbf{v} \cdot \mathbf{D}\right) W-\mathbf{v} \cdot \mathbf{E}=0, \\
D_{\nu} F^{\mu \nu}=\jmath^{\mu}=m^{2}\left\langle v^{\mu} W\right\rangle .
\end{gathered}
$$

Theory 2: $\omega \ll k \ll m$

$$
\begin{gathered}
\mathbf{v} \cdot \mathbf{D} W-\mathbf{v} \cdot \mathbf{E}=-\delta \hat{C} W+\xi \\
\langle W\rangle=0 \\
\mathbf{D} \times \mathbf{B}=\mathbf{j}=m^{2}\langle\mathbf{v} W\rangle \\
\langle\langle\xi \xi\rangle\rangle=\frac{2 T}{m^{2}} \delta \hat{C} .
\end{gathered}
$$

Theory 3: $\omega \ll k \ll \gamma$

$$
\begin{gathered}
\sigma \mathbf{E}=\mathbf{D} \times \mathbf{B}+\boldsymbol{\zeta} \\
\langle\langle\boldsymbol{\zeta} \boldsymbol{\zeta}\rangle\rangle=2 \sigma T .
\end{gathered}
$$

\footnotetext{
${ }^{6}$ The derivation in Ref. [16] treats the color charge of the charge carriers as classical, using Wong equations. This approach has been used before in analyzing hot gauge theories, and it is worth pointing out one way to understand, after the fact, why it works. In the case at hand, note that the result (5) for $\sigma$ does not depend on the color representations of the charge carriers in any way except implicitly in the value of $m^{2}$. At leading order, the contribution to $\mathrm{m}^{2}$ of any charge carrier species is proportional to the adjoint Casimir $C_{R}$ for the color representation of that species, and there is no other representation dependence. If one extracts the proportionality constant for representations with arbitrarily large values of $C_{R}$, one will then also automatically have the right proportionality constant for small representations. But the color of large representations can be treated classically. So treating the color of the charge carriers classically and then setting the square of the classical charge to the quantum value $Q^{2}=C_{R}$ at the end of the day works because of the simplicity of the color structure for the quantities of interest.
} 
All theories have been written in forms that are local in space and time. All fields should be interpreted as classical.

Theory 1. The first effective theory is a now-standard formulation of "hard-thermalloop" dynamics [19] which is valid for $\omega, k \ll T$, up to corrections suppressed by powers of $g$. These equations amount to linearized, collisionless, non-Abelian, Boltzmann-Vlasov kinetic theory. One has conceptually split the degrees of freedom in the underlying quantum field theory into those associated with quanta that have large momenta $p \sim T$ and those with low momenta $k \ll T$. The high momentum (or "hard") quanta are treated collectively by a linearized Boltzmann equation; the (bosonic) low momenta modes, referred to as "soft" (and sometimes also semi-hard) modes are, because of the high occupation numbers mentioned earlier, treated as comprising a classical field. The first equation (8a) is a collisionless linearized Boltzmann equation for the propagation of the hard quanta in the presence of the soft gauge fields. $W(\mathbf{x}, \mathbf{v}, t)$ represents the color distribution of hard particles in space, velocity, and time, where the velocity $\mathbf{v}$ is a unit vector because all hard particles are ultra-relativistic, and where $\langle\cdots\rangle$ in Eqs. (8) and (9) denotes averaging over the direction of $\mathbf{v}$. Although the hard particles have individual momenta $p \sim T$ associated with very short wavelengths, the collective distribution of hard particles, described by $W$, can have slow spatial variation (in other words, the density of hard particles may vary slowly over a large region of space), which is why $W$ appears in the effective theory describing $\omega, k \ll T$ dynamics.

The distribution $W$ appearing in the above effective theories only encodes information about the color structure of collective fluctuations, and $W$ lives in the adjoint representation of the gauge group. ${ }^{7}$ (See Refs. $[5,2,20]$ for details.) A full description of all physics for $\omega, k \ll T$ would also contain additional distribution functions describing fluctuations that do not contribute to the color current. These include color-neutral fluctuations which are responsible for hydrodynamic phenomena such as viscosity and sound, but which do not couple to the long-distance color dynamics except at higher order in the coupling $g$. We will therefore ignore them. ${ }^{8}$ For a discussion of a tower of effective theories relevant to the uncolored sector, see Ref. [21].

There are several things we have left out of Theory 1 because they do not affect color dynamics at leading order. One is a collision term in the Boltzmann equation (8a) due to hard collisions. The cross-section for such collisions is $O\left(\alpha^{2}\right)$ and is overwhelmed, for color dynamics, by softer collisions that will be discussed in the context of Theory 2 below. ${ }^{9}$ We

\footnotetext{
${ }^{7}$ Technically, $W$ is the adjoint representation piece of the density matrix describing the color charges of the hard excitations, summed over the various species of excitations and integrated over the energy of excitations (for a fixed direction of motion $\mathbf{v}$ ). It is normalized in a way that simplifies the resulting equation.

${ }^{8}$ There are also other colored sectors besides the adjoint one represented by $W$. For each species of hard
excitations, there are sectors corresponding to every irreducible representation contained in $R \times \bar{R}$, where
$R$ is the color representation of the given excitation. See Ref. [5] for a discussion. However, we do not know
of any interesting physics that specifically couples to the other colored sectors except at sub-leading order
in $g$.

${ }^{9}$ Hard collisions are relevant at leading order in $g$ to physics in the uncolored sector, however. See, for example, Ref. [22].
} 
have also left out the soft modes of non-gauge fields such as scalars or fermions. These do not affect the color conductivity or the final soft dynamics (Theory 3) at leading order in $g$, provided the effective thermal mass of any colored scalars is large enough [large compared to $\left.g^{2} T\right]$ so that they decouple at the very soft scales of interest for Theory 3 .

Theory 2. The $\omega \ll k \ll m$ limit of Theory 1 is a small frequency version of a theory originally written down by Bödeker [2], and is discussed in detail in Ref. [12]. In this low frequency regime, all time derivative terms in Theory 1 become negligible, and have been dropped, except for the one implicit in $\mathbf{E}$ (which is $-d \mathbf{A} / d t$ in $A_{0}=0$ gauge). Gauss' Law is replaced by the constraint (9b) due to the effects of Debye screening for $k \ll m$. (See Ref. [12] for details and interpretation.)

The most physically significant change is the introduction of the collision term $\delta \hat{C} W$ into the linearized Boltzmann equation (9a). This term represents the effects of $2 \rightarrow 2$ collisions of hard particles via the $t$-channel exchange of what are called semi-hard gauge bosons $(\omega \lesssim k \lesssim m)$. The leading log calculation of the linearized collision operator $\delta \hat{C}$ was first made by Bödeker [2] and is explained in the language of collisions in Ref. [5]. One finds,

$$
\begin{gathered}
\delta \hat{C} f(\mathbf{v}) \equiv\left\langle\delta C\left(\mathbf{v}, \mathbf{v}^{\prime}\right) f\left(\mathbf{v}^{\prime}\right)\right\rangle_{\mathbf{v}^{\prime}}, \\
\delta C\left(\mathbf{v}, \mathbf{v}^{\prime}\right) \approx \gamma(\mu)\left[\delta^{S_{2}}\left(\mathbf{v}-\mathbf{v}^{\prime}\right)-\frac{4}{\pi} \frac{\left(\mathbf{v} \cdot \mathbf{v}^{\prime}\right)^{2}}{\sqrt{1-\left(\mathbf{v} \cdot \mathbf{v}^{\prime}\right)^{2}}}\right],
\end{gathered}
$$

where

$$
\gamma(\mu) \approx C_{\mathrm{A}} \alpha T \ln \left(\frac{m}{\mu}\right)
$$

is the leading log contribution to the hard gauge boson damping rate due to exchange of gauge bosons having spatial momentum $q>\mu$. In other words, in a Wilsonian view of renormalization, $\mu$ is the ultraviolet cut-off imposed on $k$ to define effective theory 2 , and $\gamma(\mu)$ is the damping rate due to gauge field fluctuations which have been integrated out and are no longer present in the effective theory. (In practice, it will be more convenient to perform renormalization by standard subtraction methods, and $\mu$ will be the renormalization scale rather than an ultraviolet cut-off.) The normalization of the angular delta function $\delta^{S_{2}}(\mathbf{v})$ is given in table II.

The collision term in Theory 2 causes dissipation in the effective theory and so an appropriately matched thermal noise term must also be present. The $\xi$ in (9a) is Gaussian noise with correlation [2]

$$
\left\langle\left\langle\xi(\mathbf{v}, \mathbf{x}, t) \xi\left(\mathbf{v}^{\prime}, \mathbf{x}^{\prime}, t^{\prime}\right)\right\rangle\right\rangle=\frac{2 T}{m^{2}} \delta C\left(\mathbf{v}, \mathbf{v}^{\prime}\right) \delta\left(t-t^{\prime}\right) \delta^{(3)}\left(\mathbf{x}-\mathbf{x}^{\prime}\right) .
$$

A path integral representation of this effective theory may be found in Ref. [12].

Theory 3. This is Bödeker's effective theory, discussed earlier, and is obtained at leading log order by dropping $\mu$ below $\gamma$ (the momentum scale below which Theory 3 becomes valid) 
in Theory 2, and then solving the $W$ equations for $k \ll \gamma$ [which allows one to drop $\mathbf{v} \cdot \mathbf{D}$ compared to $\delta \hat{C}$ in (9a)]. Details may be found in Refs. [2,5]. We emphasize that, for the purpose of expanding in inverse powers of logarithms, we formally consider the momentum scale $k \sim g^{2} T$ of ultimate interest to be parametrically small compared to $\gamma \sim g^{2} T \ln \left(g^{-1}\right)$.

The decoupling of massive fields from low energy physics is very familiar in Euclidean field theory, and forms the basis for traditional applications of effective field theory techniques. In the case at hand, it is possible to eliminate the field $W$ (while maintaining a local description in the effective theory) because one is dropping below the scale $\gamma$ characterizing the decay time of color correlations. This harks back to an old suggestion by Lebedev and Smilga [23] that $\gamma$ might cut off infrared divergences for some quantities. (See also Ref. [24].) As we shall see, this indeed happens for the color conductivity at next-to-leading log order, in a sense that we will make precise.

It has long been known that the leading-order hard-thermal-loop effective interactions between gauge fields with momentum $k \sim m \sim g T$ are non-local in space and time. That is, formally eliminating $W$ from the Theory 1 Eqs. (8) generates non-local interactions for A. It should be emphasized that it is the inclusion of collisions which allows one to recover locality of effective gauge interactions for $k \ll \gamma$, as in Theory 3 . In the non-Abelian theory, arbitrarily small angle collisions will cause the color correlations of hard particles to fall off rapidly for times and distances large compared to the relevant collision time $1 / \gamma$, permitting one to treat such correlations as local in an effective theory restricted to $k \ll \gamma$. Leadingorder calculations using hard-thermal-loop interactions miss this effect because they do not incorporate the effect of collisions on the propagation of hard color fluctuations. In such a collisionless approximation, the disturbance created by probing the hard particles at one time does not decay, and can have an arbitrarily long-lasting effect on fluctuations measured at a later time-hence, non-locality. With collisions included, this non-locality is cut-off at the scale $1 / \gamma$.

\section{SCALING AND SUBLEADING CORRECTIONS}

\section{A. Review of static case}

One of the great advantages to using effective theories is that, besides simplifying the description of the relevant physics at a given scale, they provide a very clean mechanism for organizing, analyzing, and computing sub-leading corrections. For hot gauge theories, the quintessential example is the analysis of static equilibrium properties by Braaten and Nieto [25] using a sequence of Euclidean effective theories. (See also Ref. [26].) The important scales for static equilibrium physics are $T, g T$, and $g^{2} T$; the scale $\gamma$, which will be crucial to our discussion of dynamics, does not appear. Below $T$, the effective theory is a threedimensional Euclidean gauge theory coupled to an adjoint scalar $A_{0}$ with mass $m=O(g T)$, this mass being the manifestation of Debye screening. Below the scale $m$, the $A_{0}$ field decouples, and one is left with a three-dimensional theory of unscreened magnetic physics:

$$
S_{\mathrm{eff}}=T^{-1} \int d^{3} \mathbf{x}\left[\frac{1}{2} F_{i j}^{a} F_{i j}^{a}+\cdots\right] .
$$

The term shown explicitly is simply renormalizable (in fact finite) 3-dimensional pure gauge theory. The dots represent an infinite sequence of interactions with higher and higher scaling 
dimension $\left(F^{3}, F^{4}\right.$, etc). These terms are infrared irrelevant, in the sense of the renormalization group, and their importance is suppressed by higher and higher powers of the scale $k$ of the physics of interest, compared to the scale $\Lambda=m \sim g T$ where the effective theory breaks down. So, for the study of non-perturbative physics, where $k \sim g^{2} T$, terms in $S_{\text {eff }}$ with higher and higher scaling dimension correspond to corrections whose effects are suppressed by higher and higher powers of the ratio $k / m \sim g$.

It is important to note that the coefficients of specific higher order terms may also contain explicit factors of $g$, which will cause their effects to be even more suppressed than indicated by their scaling dimension alone.

Standard power counting of 3-dimensional gauge theory (14) gives the ultraviolet scaling dimension of the field $\mathbf{A}$ to be $[A]=[x]^{-1 / 2}$ (or equivalently $[k]^{1 / 2}$ ). One typically rescales the field $\mathbf{A} \rightarrow T^{-1 / 2} \mathbf{A}$ so that the coefficient of the kinetic term in (14) is dimensionless and so that counting scaling dimension is then the same as counting engineering dimension. In this paper, however, we shall avoid such redefinitions in order not to obscure the relationship between the gauge fields appearing in successive effective theories.

Braaten and Nieto showed how, in principle, one can calculate the coefficients of the higher-dimensional terms in the effective theory (14), to whatever order desired, by carrying out perturbative matching calculations. Perturbative calculations suffice because the effective theory is valid for scales $k \ll m$, and because the physics of gauge field fluctuations is perturbative at the upper end $g^{2} T \ll k \ll m$ of that range of validity. Roughly speaking, the idea is to pick a set of physical quantities that probe physics at a scale $k \ll \Lambda$ where the effective theory is valid, then to calculate those quantities in both the effective theory and the underlying theory, and then fix the (finite) set of unknown coefficients (at a given order) by requiring the answers to be the same. A refinement is required to make the calculation tractable, because long-distance physics in the effective theory (14) [and so also in the underlying theory] is non-perturbative! Conceptually, one imagines restricting the theory to a large but not too large box — one of size $L$ which is large enough $\left(L \gg \Lambda^{-1}\right)$ for the effective theory expansion to be valid, but small enough $\left(L \ll\left(g^{2} T\right)^{-1}\right)$ that all the physics in the box is perturbative. The matching calculations can then be performed perturbatively.

The "large box" temporarily introduced for the sake of matching can be replaced by any suitable infrared regularization, and in practice it is most convenient to use dimensional regularization. Dimensional regularization is also used for ultraviolet regularization, if required to define the theory (at a given order) in the first place.

\section{B. The dynamic case}

Given that our goal is to expand in inverse powers of $\operatorname{logarithms} \ln \left(g^{-1}\right)$, while always working to leading order in powers of $g$, trying to refine our first two effective theories (8) and (9) by introducing new infrared-irrelevant interactions is unnecessary. We need to be able to use Theory 1 at the scale $m$ in order to match onto Theory 2, but then irrelevant interactions are suppressed by powers of $m / T \sim g$, not by mere powers of inverse logs. We need to be able to use Theory 2 at the scale $\gamma$ in order to match onto Theory 3 , but then irrelevant interactions are suppressed by powers of $\gamma / m \sim g \ln \left(g^{-1}\right)$, which can again

be ignored. The crux of an expansion in inverse logs, as far as the introduction of higherdimensional interactions is concerned, is in the application of Theory 3 (Bödeker's effective 
theory) to non-perturbative physics at a scale $k \sim g^{2} T \ll \gamma$. The influence of higher dimension irrelevant terms will then only be suppressed by factors of $k / \gamma \sim 1 / \ln \left(g^{-1}\right)$.

Fortunately, a delightful simplification occurs, to be explained momentarily: effects of adding any higher-dimension corrections to Bödeker's effective theory (10) are suppressed by more than one power of the inverse logarithm. This means that Bödeker's effective theory is already perfectly adequate at next-to-leading log order, provided one calculates its one parameter (namely $\sigma$ ) to next-to-leading log order. Previous numerical simulations of Bödeker's theory [3] can therefore be instantly extended to NLLO accuracy simply by using the improved value of $\sigma$.

Here is one version of the power counting analysis which demonstrates the required suppression of higher-dimension operators. It is possible to turn the Langevin equation (10) that defines the effective theory into a supersymmetric path integral form that allows standard renormalization analysis [12], which we review in the appendix. But one can get to the same result in a simpler, more cavalier way. The dynamical effective theory has to (and does) produce the same equilibrium distribution that is more traditionally analyzed with the Euclidean effective theories discussed earlier. We can therefore borrow the well-known result that the field $\mathbf{A}$ scales with distance as $[A]=[x]^{-1 / 2}$ in the ultraviolet. That means that, at short distance, $\mathbf{D} \rightarrow \boldsymbol{\nabla}$ and Bödeker's effective theory becomes the free theory $\sigma \mathbf{E}=\boldsymbol{\nabla} \times \mathbf{B}+\boldsymbol{\zeta}$. This is just $-\sigma d \mathbf{A} / d t=\boldsymbol{\nabla} \times \boldsymbol{\nabla} \mathbf{A}+\boldsymbol{\zeta}$ in $A_{0}=0$ gauge, and implies that time scales as $[t]=[x]^{2}$. This, of course, is just a re-iteration of (3). The lowest dimension terms that could possibly be added to the equation (10) for the effective theory, consistent with parity and gauge-invariance, are $\mathbf{E} \times \mathbf{B}$ and $\mathbf{B} \times \mathbf{D} \times \mathbf{B}$. These terms have scaling dimension $[x]^{-4}$ as opposed to those in Bödeker's equation (10), which are $[x]^{-5 / 2}$. The difference of $3 / 2$ in scaling dimension means their effect is suppressed ${ }^{10}$ by $(k / \gamma)^{3 / 2}$. So, based simply on scaling dimension alone, the effect of possible higher dimension operators, when $k=O\left(g^{2} T\right)$, is suppressed by at least $\left[\ln \left(g^{-1}\right)\right]^{-3 / 2}$. This substantiates our claim that the form of Bödeker's equation (10) remains unchanged to next-to-leading log order. The effects of higher dimension terms may, of course, be even smaller that this estimate if there is explicit suppression in their coefficients. In fact, as discussed in the appendix, the coefficients of these operators necessarily include a factor of $g$ which implies a further suppression of $\left(g^{2} T / \gamma\right)^{1 / 2}$. Hence, at the $g^{2} T$ scale, the actual relative correction due to higher-dimension operators is at least $\left[\ln \left(g^{-1}\right)\right]^{-2}$.

\section{Implications for the meaning of $\sigma$}

The above discussion allows us to provide an unambiguous definition of what we mean by the color conductivity at NLLO: we simply define it to be the coefficient of Bödeker's effective theory (10) at that order. This is a non-trivial issue because the conventional definition of conductivity for an Abelian gauge theory, in terms of the linear response of the

\footnotetext{
${ }^{10}$ It should be noted that this power counting argument gives the correct suppression factor when comparing terms that are either infrared irrelevant or marginal, and which do not have unnaturally small coefficients at the scale of the cutoff on the effective theory. It is the derivative terms in the uncorrected effective theory which define marginal scaling.
} 
current $\mathbf{j}$ to a static, homogeneous electric field $\mathbf{E}$, does not generalize in a meaningful way to a non-Abelian theory. This is because, in a non-Abelian theory, there is no gauge-invariant way to define the induced current $\mathbf{j}$, nor is there any straightforward way to define the imposition of a "homogeneous" background electric field on top of the thermally fluctuating gauge field. ${ }^{11}$ In contrast, Bödeker's effective theory is a local theory, and Eq. (10) is nicely gauge covariant with $\sigma$ a gauge-invariant numerical constant. ${ }^{12}$

Beyond NLLO, however, the definition of $\sigma$ as the parameter of the effective theory presumably becomes ambiguous. That's because at next-to-next-to-leading log order (NNLLO), we need to introduce irrelevant infrared interactions into the theory, namely $\mathbf{E} \times \mathbf{B}$ and $\mathbf{B} \times \mathbf{D} \times \mathbf{B}$, as discussed earlier. Such interactions render the theory non-renormalizable and so require the introduction of UV regularization to what was previously a UV-finite effective theory. That is entirely normal for an effective theory, but it means that the parameters of the theory will now be convention dependent, depending on the arbitrary choices of renormalization scheme and renormalization scale. So, barring a conspiracy, we only have a clean, unambiguous definition of $\sigma$ at NLLO. It is entirely possible that color conductivity is only an approximate concept and that there is no natural, unambiguous definition beyond this order.

An unambiguously defined conductivity is, of course, irrelevant to the success of the effective theory at any order, just as (the lack of) a convention-independent coupling constant is irrelevant to the applicability of familiar zero-temperature perturbation theory.

\section{THE RESULT FOR $\sigma$}

The details of our calculation of NLLO color conductivity are given in Ref. [13], but we will summarize the main results here. Throughout, we use dimensional regularization in $d=3-\epsilon$ spatial dimensions with gauge coupling $\mu^{\epsilon / 2} g$.

\section{A. Matching Theory 1 to Theory 2}

Matching Theory 1 to Theory 2 involves calculating, to the requisite accuracy, the linearized collision operator $\delta \hat{C}$ which results from the effects of gauge field fluctuations in Theory 1, in the presence of a perturbative infrared cut-off. Conceptually, one must then repeat this calculation in Theory 2 taking into account both the bare collision operator in Theory 2, and the residual effects of gauge field fluctuations which remain in Theory 2. A major virtue of using dimensional continuation for both infrared and ultraviolet regulariza-

\footnotetext{
${ }^{11}$ Actually, one can give a sensible meaning to a uniform non-Abelian gauge field [27]. But this does not generalize in a natural way to defining the addition of a uniform external field on top the thermal fluctuations in the gauge field.

${ }^{12}$ The alert reader may object that we earlier expressed uncertainty as to whether Eq. (10) is correct (and unambiguous) in gauges other than flow gauges. However, the Langevin equation (10) can be reformulated as a manifestly gauge-invariant path integral, as discussed in Ref. [12].
} 
tion is that, with this choice of regulator, the total collision operator in Theory 2 equals the bare operator; the residual gauge field fluctuations in Theory 2 have no net effect.

The computation in Theory 1 may be carried out using any of the approaches that have been used for leading log results $[2,5,3,16]$. The kernel of the collision operator is given by

$$
\delta C\left(\mathbf{v}, \mathbf{v}^{\prime}\right)=\frac{C_{\mathrm{A}} m^{2} T}{2 g^{2} \mu^{\epsilon}}\left[\left\langle\int_{\mathbf{q}}\left|\mathcal{M}\left(\mathbf{v}, \mathbf{v}^{\prime}, \mathbf{q}\right)\right|^{2}\right\rangle_{\mathbf{v}^{\prime}} \delta^{S_{2}}\left(\mathbf{v}-\mathbf{v}^{\prime}\right)-\int_{\mathbf{q}}\left|\mathcal{M}\left(\mathbf{v}, \mathbf{v}^{\prime}, \mathbf{q}\right)\right|^{2}\right],
$$

with the scattering amplitude $\mathcal{M}$ which results from $t$-channel gauge boson exchange with hard-thermal-loop (HTL) self-energies included,

$$
\int_{\mathbf{q}}|\mathcal{M}|^{2}=g^{4} \mu^{2 \epsilon} \int \frac{d^{4-\epsilon} Q}{(2 \pi)^{4-\epsilon}}\left|v_{\mu}\left(\frac{P_{\mathrm{T}}^{\mu \nu}(Q)}{Q^{2}+\Pi_{\mathrm{T}}(Q)}+\frac{P_{\mathrm{L}}^{\mu \nu}(Q)}{Q^{2}+\Pi_{\mathrm{L}}(Q)}\right) v_{\nu}^{\prime}\right|^{2} 2 \pi \delta(Q \cdot v) 2 \pi \delta\left(Q \cdot v^{\prime}\right) .
$$

( $P_{\mathrm{T}}$ and $P_{\mathrm{L}}$ are transverse and longitudinal projection operators, respectively. See Ref. [13] for details.) The resulting form of the kernel $\delta C\left(\mathbf{v}, \mathbf{v}^{\prime}\right)$, accurate to NLLO, is much more complicated that the leading-log expression (11b). Fortunately, the only portion of $\delta C$ which is actually needed for the determination of the NLLO color conductivity is the single matrix element

$$
\gamma_{1} \equiv\left\langle v^{i} \delta \hat{C} v^{i}\right\rangle_{\mathbf{v}}
$$

At leading-log order, this is the same as the hard gauge boson damping rate (6), but at NLLO these quantities differ. We find [13] that to leading order in $g$ and all orders in $1 / \ln \left(g^{-1}\right)$,

$$
\gamma_{1}(\mu)=C_{\mathrm{A}} \alpha T\left[-\frac{1}{\epsilon}+\ln \left(\frac{m}{\bar{\mu}}\right)+\frac{1}{2} \ln \frac{\pi}{4}+a_{1}\right]
$$

where we have written the result in terms of the $\overline{\mathrm{MS}}$ scale $\bar{\mu}=\sqrt{4 \pi} e^{-\left(\gamma_{\mathrm{E}} / 2\right)} \mu$. The constant $a_{1}$ is given by the integral

$$
a_{1} \equiv \frac{1}{4} \int_{-1}^{+1} d \lambda\left\{\left(1-\lambda^{2}\right)\left[\frac{1}{2} \frac{\arg \rho_{\mathrm{T}}(\lambda)}{\operatorname{Im} \rho_{\mathrm{T}}(\lambda)}+\frac{\arg \rho_{\mathrm{L}}(\lambda)}{\operatorname{Im} \rho_{\mathrm{L}}(\lambda)}+\operatorname{Re} \frac{\ln \rho_{\mathrm{T}}^{*}(\lambda)-\ln \rho_{\mathrm{L}}(\lambda)}{\rho_{\mathrm{T}}^{*}(\lambda)-\rho_{\mathrm{L}}(\lambda)}\right]-\frac{1}{|\lambda|}\right\} .
$$

with

$$
\begin{aligned}
& \rho_{\mathrm{L}}(\lambda)=1-\frac{\lambda}{2} \ln \left(\frac{1+\lambda}{1-\lambda}\right)+\frac{i \pi}{2} \lambda, \\
& \rho_{\mathrm{T}}(\lambda)=\frac{1}{2}\left[\frac{1}{1-\lambda^{2}}-\rho_{\mathrm{L}}(\lambda)\right] .
\end{aligned}
$$

(A more explicit integral representation for $a_{1}$ may be found in [13].) Numerically,

$$
a_{1}=0.323833 \cdots
$$

As noted above, the corresponding calculation in Theory 2, when dimensionally regularized, is trivial, and matching Theory 2 to Theory 1 merely means setting the collision 
operator equal to the result of (15) and (16), with the same matrix element $\gamma_{1}$ appearing in (18). This is the bare value of $\gamma_{1}$ in Theory 2 ; to obtain the renormalized value in minimal subtraction, simply drop the $1 / \epsilon$ pole.

It should be emphasized that our argument that the color conductivity $\sigma$ can be unambiguously defined at NLLO as the parameter of an effective theory does not apply to $\gamma_{1}$. Because Theory 2 is not ultraviolet finite, its bare parameters depend explicitly on the renormalization scale $\mu$, as seen explicitly in the above result for $\gamma_{1}(\mu)$. This is completely analogous to, for example, the minimal-subtraction definition of quark mass in QCD. But a precise, convention independent definition of $\gamma_{1}$ is irrelevant (and unnecessary) for the correct evaluation of physical quantities. ${ }^{13}$

\section{B. Matching Theory 2 to Theory 3}

Matching Theory 2 to Bödeker's effective theory at NLLO requires a one-loop calculation in both these theories. In Ref. [13], we describe how appropriate ratios of large time-like Wilson loops may be chosen as the physical quantities whose expectations are matched in order to determine the parameter $\sigma$ of Bödeker's effective theory. In practice, this is completely equivalent to matching the $\omega \ll k, k \rightarrow 0$ behavior of the $A_{0}$ self-energy $\Pi_{00}(\omega, k)$ in Coulomb gauge. We find,

$$
\sigma=\frac{m^{2}}{d \gamma_{1}}\left\{1-\frac{C_{\mathrm{A}} \alpha T}{\gamma}\left[\frac{1}{\epsilon}+\ln \left(\frac{\bar{\mu}}{\gamma}\right)+b\right]+O\left(\frac{1}{\ln ^{2}(1 / g)}\right)\right\}
$$

where $\bar{\mu}$ is again the $\overline{\mathrm{MS}}$ scale, and the constant $b$ is given by

$$
b=\frac{2}{\pi} \int_{0}^{\infty} d \rho\left[\frac{1}{2}\left(\Sigma_{0}(\rho)-\frac{3}{\rho^{2}}\right)+\frac{8}{3 \rho^{2}}\left(1-\Sigma_{1}(\rho)\right)+\frac{4}{15} \Sigma_{2}(\rho)-\frac{\pi \rho}{2\left(\rho^{2}+1\right)}\right] .
$$

Here, $\rho$ represents the dimensionless magnitude $|\mathbf{p}| / T$ of a loop momentum, and $\Sigma_{m}(\rho)$ are the dimensionless functions defined in bra-ket notation as

$$
\Sigma_{m}(\rho)=\left\langle m m\left|\left(i v_{z} \rho+\delta \hat{C} / \gamma\right)^{-1}\right| m m\right\rangle,
$$

where $\delta \hat{C}$ is the linear operator defined by (11), the inverse is a non-trivial operator inverse (because $\delta \hat{C}$ and $v_{z}$ do not commute), and $|l m\rangle$ represents the function $Y_{l m}(\mathbf{v})$. We give formulas for evaluating these functions numerically in Ref. [13]. The numerical result for $b$ is

$$
b=2.8380 \cdots
$$

\footnotetext{
${ }^{13}$ The matrix element $\gamma_{1}$ differs by a finite regularization independent amount from the hard gauge boson damping rate $[13,24]$. Many attempts have been made to formulate a gauge-invariant "pole mass" definition of the damping rate for non-Abelian theories, but these have so far led to a mire of confusion [29]. For discussion of the related (but quite distinct) issue in QED, see Ref. [30].
} 


\section{Final result}

Combining Eqs. (18) and (22) yields our NLLO result for the color conductivity. The structure is clearest if we write an expansion for $\sigma^{-1}$ (the "color resistivity") rather than $\sigma$ directly. One finds

$$
\sigma^{-1}=\frac{3 C_{\mathrm{A}} \alpha T}{m^{2}}\left[\ln \left(\frac{m}{\gamma(\mu)}\right)+\mathcal{C}+O\left(\frac{1}{\ln (1 / g)}\right)\right],
$$

with

$$
\mathcal{C} \equiv \frac{1}{2} \ln \frac{\pi}{4}+a_{1}+b=3.0410 \cdots
$$

Note that the $1 / \epsilon$ divergences have canceled, as they must. Inside the logarithm of (26), $\gamma(\mu)$ is to be understood as simply the leading-log formula

$$
\gamma(\mu) \approx C_{\mathrm{A}} \alpha T \ln \left(\frac{m}{\mu}\right)
$$

and $\mu$ should be chosen so that it is of order $\gamma$. One may easily verify that the $\mu$ dependence in the NLLO result (26) only affects that answer at order $[\ln (m / \gamma)]^{-1} \sim[\ln (1 / g)]^{-1}$, which is beyond the order of this calculation.

In order to use the NLLO result (26) in any practical calculation one must, of course, choose some particular value of $\mu$ [and ignore the unknown $O\left(1 / \ln g^{-1}\right)$ corrections]. In the absence of a full next-to-next-to-leading-log analysis, there is no clearly preferred procedure for determining an "optimal" value of $\mu$. However, one reasonably natural choice is the "fastest apparent convergence" (FAC) scheme ${ }^{14}$ which is to choose $\mu$ so that the next-toleading order correction vanishes. In the present context, this amounts to choosing the scale $\mu_{\mathrm{FAC}}$ which satisfies

$$
\mu_{\mathrm{FAC}}=e^{-\mathcal{C}} \gamma\left(\mu_{\mathrm{FAC}}\right)
$$

For this choice, the NLLO conductivity is simply

$$
\sigma_{\mathrm{NLLO}}^{-1}=\frac{3 C_{\mathrm{A}} \alpha T}{m^{2}} \ln \left(\frac{m}{\mu_{\mathrm{FAC}}}\right) .
$$

\section{CONCLUSION}

We have shown that at next-to-leading log order, no modification is required to Bödeker's effective theory for non-perturbative color dynamics other than the insertion of the correct NLLO value of the color conductivity. Using our NLLO result (26), one may instantly

\footnotetext{
${ }^{14}$ At our next-to-leading-log order, one cannot use any minimal sensitivity criterion, since the NLLO result (26) has no stationary point in $\mu$.
} 
generalize Moore's numerical result (7) for the topological transition rate of hot electroweak theory to NLLO:

$$
\Gamma \approx(10.8 \pm 0.7)\left(\frac{g T}{m}\right)^{2} \alpha_{\mathrm{w}}^{5} T^{4}\left[\ln \left(\frac{m}{\gamma(\mu)}\right)+3.041+O\left(\frac{1}{\ln (1 / g)}\right)\right]
$$

Moore, and others, have also obtained numerical results for the topological transition rate by using a more microscopic theory (analogous to a lattice version of what we called Theory 1) [31,32]. Moore also attempted to estimate the size of the NLLO correction to $\Gamma$ by fitting the results of these simulations to the functional form

$$
\Gamma=\kappa\left(\frac{g T}{m}\right)^{2} \alpha_{\mathrm{w}}^{5} T^{4}\left[\ln \left(\frac{m}{g^{2} T}\right)+\delta\right],
$$

with the value of $\kappa$ fixed to 10.8, as determined from simulations of Bödeker's effective theory. This led to $\delta \approx 3.6$, with perhaps $20 \%$ uncertainty due to systematic errors [32]. ${ }^{15}$ This implies an estimate of 4.4 for the value of the $\ln \left(m / g^{2} T\right)+\delta$ factor in (31), which may be compared with the square bracket appearing in (30). This comparison is presented graphically in Fig. 1. The solid line is a plot of $\ln [m / \gamma(\mu)]+\mathcal{C}$ as a function of $\gamma / \mu$, for the specific case of electroweak theory with a single Higgs doublet and $g^{2}=0.4$. The dashed line indicates the value of 4.4 estimated in [32]. The arrow on the abscissa indicates the FAC point, where $\gamma(\mu) / \mu=e^{\mathcal{C}} \simeq 20.93$.

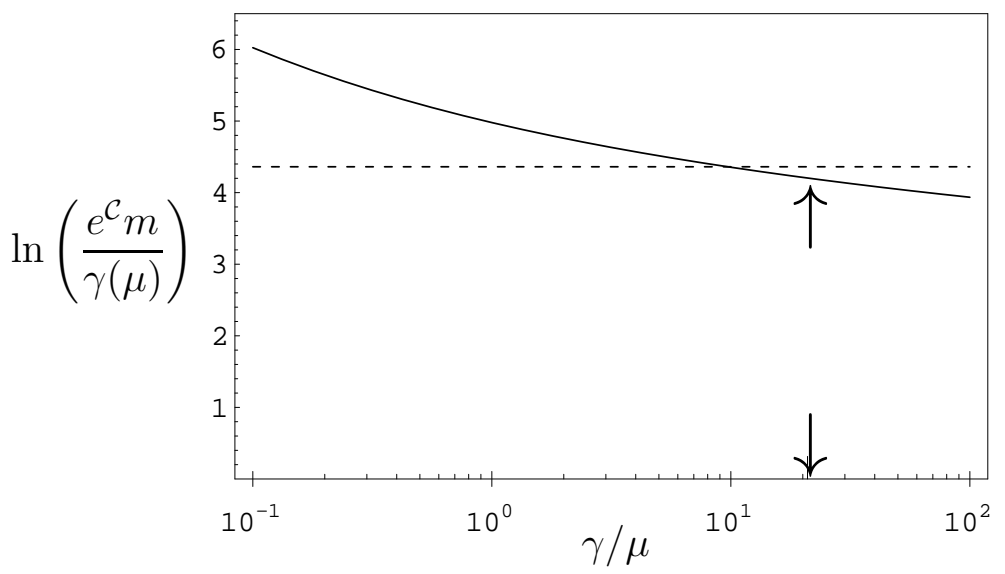

FIG. 1. The value of the $\ln [m / \gamma(\mu)]+\mathcal{C}$ factor, appearing in the inverse color conductivity (26) and in the topological transition rate (30), plotted as a function of $\gamma / \mu$, for electroweak theory with a single Higgs doublet and $g^{2}=0.4$. The dashed line indicates the value of 4.4 for which the NLLO result for the topological transition rate (30) (with the unknown yet-higher-order $1 / \ln g^{-1}$ terms neglected) agrees with independently determined results from more microscopic numerical simulations [31,32]. This value should be regarded as having a significant systematic uncertainty of perhaps $20 \%$. The arrow on the abscissa indicates the FAC point where $\gamma(\mu) / \mu=e^{\mathcal{C}} \simeq 20.93$.

\footnotetext{
${ }^{15}$ Lattice artifacts exist in these more microscopic simulations (due, in particular, to the lattice dispersion relation allowing unwanted Cherenkov radiation), which cause them not to reproduce, precisely, the dynamics responsible for NLLO corrections to $\Gamma$. These effects have only been crudely estimated, but are a major part of the uncertainty in the estimate of $\delta$.
} 
The similarity between our NLLO result and the value inferred from numerical simulations is remarkable. There was no obvious reason a priori why it should be a reasonable approximation to treat logarithms of the gauge coupling [that is, $\ln (\# / g)$ ], as large for physical values of the coupling. However, one sees from Fig. 1 that even the most naive prescription of picking $\mu$ exactly equal to the scale $\gamma$ produces a result within $20 \%$ of Moore's numerical estimate (which itself has an uncertainty estimated at 20\%). The naive prescription of letting $\mu$ vary by an entire order of magnitude about $\gamma$ (from $0.1 \gamma$ to $10 \gamma$ ), and taking the variation as a crude estimate of the uncertainty from leaving out higher-order corrections, still keeps the result within roughly 35\%. The FAC value of $\gamma(\mu) / \mu$ suggests that perhaps one should pay more attention to the range $\mu<\gamma$, where the variation with $\mu$ is even smaller and the agreement with Moore's numerical estimate even better.

The close agreement between the FAC value of $\mu$ and the precise point where the curves of Fig. 1 cross is striking, but probably fortuitous given the uncertainty in the numerical simulation value. Nevertheless, perhaps the characteristic size of neglected corrections really is $e^{-\mathcal{C}} \gamma$, and not just $\gamma$ (as one might naively expect). If true, this would mean that (for electroweak theory) the expansion in inverse logs actually has a respectably small expansion parameter of about 0.25. To confirm (or refute) this expectation, one would need to calculate to yet higher order in the expansion in inverse logarithms. As discussed earlier, this presumably requires adding new operators to Bödeker's effective theory, and this in turn implies that new numerical simulations would be required. Such additional higher-dimension

operators will also cause the effective theory to no longer be UV finite, implying that a careful matching calculation for the lattice-regularized theory would now be required.

\section{ACKNOWLEDGMENTS}

We thank Guy Moore and Dietrich Bödeker for a variety of helpful discussions. This work was supported, in part, by the U.S. Department of Energy under Grant Nos. DEFG03-96ER40956 and DE-FG02-97ER41027. 


\section{APPENDIX A: POWER COUNTING WITH FUNCTIONAL INTEGRALS}

In the main text, we discussed how to count the relative scaling dimensions of operators appearing in the Langevin equation. We swept under the rug issues of how to do the same for terms involving the noise. To be systematic, it is much more convenient to recast the problem in terms of a functional integral, where dimension counting of operators is more familiar. Also, in the functional integral version, noise has been integrated over, and need not be discussed separately.

The functional integral representation of Bödeker's theory is well known as the functional integral representing stochastically quantized 3-dimensional non-Abelian gauge theory. See, for example, Ref. [33] for a review, or [12] for a discussion in the present context. In $A_{0}=0$ gauge, one form is

$$
Z=\int[\mathcal{D} \mathbf{A}(\mathbf{x}, t)] J[\mathbf{A}] e^{-S[\mathbf{A}]}
$$

with

$$
S=\frac{1}{4 \sigma T} \int d t d^{3} x\left|\sigma \frac{d \mathbf{A}}{d t}+\mathbf{D} \times \mathbf{B}\right|^{2},
$$

and where $J[\mathbf{A}]$ is a Jacobian factor that equals one in dimensional regularization. It is useful to rewrite the action in terms of the magnetic energy density,

$$
\mathcal{V}(\mathbf{A}) \equiv \frac{1}{2} \mathbf{B}^{a} \cdot \mathbf{B}^{a}
$$

as

$$
S=\frac{1}{4 \sigma T} \int d t d^{3} x\left|\sigma \frac{d \mathbf{A}}{d t}+\frac{d \mathcal{V}}{d \mathbf{A}}\right|^{2} .
$$

There is a hidden supersymmetry of this functional integral which restricts the form of interactions which can be added to it. The functional integral can be rewritten in superfield notation as

$$
Z=\int[\mathcal{D} \boldsymbol{\Phi}] e^{-S[\boldsymbol{\Phi}]}
$$

with

$$
S=\frac{1}{T} \int d \theta d \bar{\theta} d t d^{3} x\left[\sigma \overline{\mathcal{D}}_{t} \boldsymbol{\Phi} \cdot \mathcal{D}_{t} \boldsymbol{\Phi}+\mathcal{V}(\boldsymbol{\Phi})\right]
$$

where $\boldsymbol{\Phi}$ is the superfield

$$
\Phi=\mathbf{A}+\bar{\theta} \mathbf{c}+\overline{\mathbf{c}} \theta+\boldsymbol{\lambda} \bar{\theta} \theta .
$$

The supersymmetry only involves time, rather than space-time, and the SUSY time derivatives are

$$
\overline{\mathcal{D}}_{t} \equiv \partial_{\theta}, \quad \mathcal{D}_{t} \equiv \partial_{\bar{\theta}}-\theta \partial_{t}
$$


In (A6), $\boldsymbol{\lambda}$ is an auxiliary field, and $\mathbf{c}$ and $\overline{\mathbf{c}}$ are ghost fields, the integral over which produces the Jacobian factor $J[\mathbf{A}]$ mentioned earlier.

The scaling dimensions of all the fields may now easily be read off from the supersymmetric action: $[t]=[x]^{2},[\theta]=[\bar{\theta}]=[x]$, and $[\boldsymbol{\Phi}]=[x]^{-1 / 2}$. (Keep in mind that for Grassmann integration, $d \theta$ has the inverse dimension of $\theta$, and so $[d \theta]=[d \bar{\theta}]=[x]^{-1}$.) One may now analyze the dimensions of what possible irrelevant interactions can be added to the supersymmetric action (A5) consistent with gauge invariance, parity, and supersymmetry. ${ }^{16}$ This provides a more familiar way to do the power counting analysis than that of the main text. For instance, consider the interaction $\mathbf{B} D^{2} \mathbf{B}$, where $\mathbf{B}$ and $D$ are to be understood as the normal non-SUSY expressions for the magnetic field and the gauge-covariant derivative, but with $\mathbf{A}$ replaced by the superfield $\boldsymbol{\Phi}$. The interaction $\mathbf{B} D^{2} \mathbf{B}$ has scaling dimension $[x]^{-5}$, as opposed to the other terms in the supersymmetric Lagrangian, which have the marginal scaling $[x]^{-3}$. So the effects of this term should be suppressed by at least $(k / \gamma)^{2}$, since $\gamma$ serves as the cut-off scale $\Lambda$ for this theory. Another example is $\overline{\mathcal{D}}_{t} \boldsymbol{\Phi} \cdot \mathcal{D}_{t} \boldsymbol{\Phi} \times \mathbf{B}$ (still keeping to $A_{0}=0$ gauge), which has scaling dimension $[x]^{-9 / 2}$, and so is suppressed by $(k / \gamma)^{3 / 2}$. In fact, the coefficient of this operator must also contain a factor of $g$, because the underlying theory is unchanged if $g \rightarrow-g$ and $\mathbf{A} \rightarrow-\mathbf{A}$ (or $\boldsymbol{\Phi} \rightarrow-\boldsymbol{\Phi}$ ). The effective theory must also respect this invariance. However a suitable rescaling of variables in Theory 2 (see Ref. [13]) shows that the theory only depends on the scale $\gamma$ plus the dimensionless combination $g^{2} T / \gamma$. Hence, a factor of $g$ in the coefficient of an induced higher dimension operator implies that the effects of this operator will have an additional suppression by $\left(g^{2} T / \gamma\right)^{1 / 2}$. Therefore, the effects of the interaction $\overline{\mathcal{D}}_{t} \boldsymbol{\Phi} \cdot \mathcal{D}_{t} \boldsymbol{\Phi} \times \mathbf{B}$ must be suppressed by $\left(g^{2} T / \gamma\right)^{1 / 2}(k / \gamma)^{3 / 2}$.

A little thought shows that the net result is that the only interactions which can be added to the action (A5), consistent with its symmetries, are either (i) irrelevant operators whose effects, at the scale $g^{2} T$ are suppressed by at least two powers of $g^{2} T / \gamma$, or (ii) total derivatives [such as $\left(\mathcal{D}_{t} \boldsymbol{\Phi}\right) \cdot \mathbf{D} \times \mathbf{B}=\mathcal{D}_{t} \mathcal{V}(\boldsymbol{\Phi})$ ] which have no effect on the dynamics.

\footnotetext{
${ }^{16}$ Although it is not required for our discussion, it is worth noting that time-reversal invariance also constrains the possible irrelevant interactions which may appear in the effective theory. This may be surprising at first sight, since the Langevin equation (10) defining the leading-order effective theory is dissipative and manifestly violates time reversal invariance. Nevertheless, this theory generates time-dependent equilibrium correlation functions which do respect time-reversal symmetry. Formally, this is easiest to see from the functional integral (A1). The action density appearing in (A1b) is not invariant under time reversal. However, after multiplying out the square, it is only the cross term which is time reversal odd. And this cross term is a total time derivative, $\int d^{3} x \sigma(d \mathbf{A} / d t) \cdot \mathbf{D} \times \mathbf{B}=(d / d t) \int d^{3} x \sigma \mathcal{V}(\mathbf{A})$. Hence, the action $(\mathrm{A} 1)$ is in fact time-reversal invariant up to boundary terms at $t= \pm \infty$, which are irrelevant as far as equilibrium properties (including time-dependent correlation functions) are concerned. This invariance (up to boundary terms) must remain true in the presence of higher-dimension irrelevant interactions. For related discussion in terms of the Fokker-Planck equation, see Ref. [34].
} 


\section{REFERENCES}

[1] For reviews, see A. Cohen, D. Kaplan and A. Nelson, Annu. Rev. Nucl. Part. Sci. 43, 27 (1993); V. Rubakov and M. Shaposhnikov, hep-ph/9603208, Usp. Fiz. Nauk 166, 493 (1996) [Phys. Usp. 39, 461 (1996)]; M. Trodden, hep-ph/9803479, Case Western report no. CWRU-P6-98.

[2] D. Bödeker, hep-ph/9810430, Phys. Lett. B426, 351 (1998); hep-ph/9905239; hep-ph/9903478.

[3] G. Moore, hep-ph/9810313.

[4] K. Kajantie, M. Laine, K. Rummukainen, and M. Shaposhnikov, hep-ph/9605288, Phys. Rev. Lett. 77, 2887 (1996). S. Elitzur, Phys. Rev. D 12, 3978 (1975).

[5] P. Arnold, D. Son, and L. Yaffe, hep-ph/9810216, Phys. Rev. D 59, 105020 (1999); hep-ph/9901304, Phys. Rev. D 60, 025007 (1999).

[6] T. Appelquist and R. Pisarski, Phys. Rev. D 23, 2305 (1981); S. Nadkarni, Phys. Rev. D 27, 917 (1983); Phys. Rev. D 38, 3287 (1988); Phys. Rev. Lett. 60, 491 (1988); N. Landsman, Nucl. Phys. B322, 498 (1989); K. Farakos, K. Kajantie, M. Shaposhnikov, Nucl. Phys. B425, 67 (1994).

[7] A. Selikhov and M. Gyulassy, nucl-ph/9307007, Phys. Lett. B316, 373 (1993).

[8] P. Arnold, D. Son, and L. Yaffe, hep-ph/9609481, Phys. Rev. D 55, 6264 (1997). See also P. Huet and D. Son, hep-ph/9610259, Phys. Lett. B393, 94 (1997); P. Arnold, hep-ph/9701393, Phys. Rev. D 55, 7781 (1997).

[9] D. Grigorev and V. Rubakov, Nucl. Phys. B299, 67 (1988); D. Grigorev, V. Rubakov, and M. Shaposhnikov, Nucl. Phys. B326, 737 (1989); for a review, see E. Iancu, hep-ph/9807299, Saclay report no. SACLAY-T98-073.

[10] H. Chan and M. Halpern, Phys. Rev. D 33, 540 (1985).

[11] J. Zinn-Justin and D. Zwanziger, Nucl. Phys. B295 [FS21], 297 (1988).

[12] P. Arnold, An effective theory for $\omega \ll k \ll g T$ color dynamics in hot non-Abelian plasmas, hep-ph/9912307.

[13] P. Arnold and L. Yaffe, "High temperature color conductivity at next-to-leading log order," hep-ph/9912306.

[14] For a review, see P. Damgaard and H. Huffel, Phys. Rept. 152, 227 (1987).

[15] R. Pisarski, Phys. Rev. Lett. 63, 1129 (1989); Phys. Rev. D 47, 5589 (1993).

[16] D. Litim and C. Manuel, hep-ph/9902430, Phys. Rev. Lett. 82, 4981 (1999).

[17] M. Basagoiti, hep-ph/9903462.

[18] J.-P. Blaizot and E. Iancu, hep-ph/9903389, Nucl. Phys. B557, 183 (1999).

[19] E. Braaten and R. Pisarski, Phys. Rev. D 45, 1827 (1992); Nucl. Phys. B337, 569 (1990); S. Mrówczyński, Phys. Rev. D 39, 1940 (1989); H.-Th. Elze and U. Heinz, Phys. Rept. 183, 81 (1989); J. Blaizot and E. Iancu, Nucl. Phys. B417, 609 (1994); and references therein.

[20] E. Iancu, hep-ph/9710543, Phys. Lett. B435, 152 (1998).

[21] P. Arnold and L. Yaffe, hep-ph/9709449, Phys. Rev. D 57, 1178 (1998).

[22] H. Heiselberg, hep-ph/9401309, Phys. Rev. D 49, 4739 (1994).

[23] V. Lebedev and A. Smilga, Phys. Lett. 253B, 231 (1991); Ann. Phys. (NY) 202, 229 (1990); Physica A181, 187 (1992).

[24] J. Blaizot and E. Iancu, hep-ph/9906485.

[25] E. Braaten and A. Nieto, Phys. Rev. D 51, 6990 (1995); 53, 3421 (1996). 
[26] K. Kajantie, M. Laine, K. Rummukainen, M. Shaposhnikov, Nucl. Phys. B458, 90 (1996).

[27] L. Brown and W. Weisberger, Nucl. Phys. B157, 285 (1979).

[28] H. Weldon, Phys. Rev. D 26, 1394 (1982); U. Heinz, Ann. Phys. (N.Y.) 161, 48 (1985); 168, 148 (1986).

[29] S. Peigné, E. Pilon, D. Schiff, Z. Phys. C60, 455 (1993); R. Baier, H. Nakkagawa, A. Niegawa, Can. J. Phys. 71, 205 (1993); T. Altherr, E. Petitgirard, T. del Rio Gaztellurutia, Phys. Rev. D 47, 703 (1993);

[30] J. Blaizot and E. Iancu, Phys. Rev. D 55, 973 (1997); 56, 7877 (1997); Phys. Rev. Lett. 76, 3080 (1996); K. Takashiba, Int. J. Mod. Phys. A11, 2309 (1996); D. Boyanovsky and J. de Vega, Phys. Rev. D 59, 105019 (1999).

[31] G. Moore, C. Hu, B. Muller, hep-ph/9710436, Phys. Rev. D 58, 045001 (1998);

G. Moore and K. Rummukainen, hep-ph/9906259; D. Bödeker, G. Moore and K. Rummukainen, hep-ph/9907545, hep-lat/9909054.

[32] G. Moore, hep-ph/9902464.

[33] J. Zinn-Justin, Quantum Field Theory and Critical Phenomena, 2nd edition (Oxford University Press, 1993).

[34] P. Arnold, "Langevin equations with multiplicative noise: resolution of time discretization ambiguities for equilibrium systems," hep-ph/9912208. 